\title{
Modeling and analysis of motion of a spacecraft with a tether aerodynamic stabilizer
}

\author{
D. Elenev ${ }^{1}$, Y. Zabolotnov ${ }^{1}$ \\ ${ }^{I}$ Samara National Research University, 34 Moskovskoe Shosse, 443086, Samara, Russia
}

\begin{abstract}
Space tether system consists of two solid bodies connected by a tether. The deployment of this system is produced mainly by aerodynamic forces which act on the bodies. One of these bodies has higher ballistic coefficient thus acting as an aerodynamic stabilizer on low orbit. Such tether systems allow to lower the requirements for characteristics of a spacecraft and can be used for purposes of stabilization in higher layers of the atmosphere, for utilization of space debris. For mathematical modeling purposes the tether is represented as a set of mathematical points with elastic connections.
\end{abstract}

Keywords: tether system; stabilization; spacecraft; deployment; multipoint model of the tether

\section{Introduction}

The modeling of the motion of a spacecraft with a tether aerodynamic stabilizer is made on the atmosphere part of the motion during the deployment of the tether system. The tether system consists of a spacecraft, a stabilizer, and a tether. The deployment and orientation of the tether system are produced by aerodynamic forces. The stabilizer compared to spacecraft has a high ballistic coefficient $\sigma=C_{x v} S / m$, where $C_{x v}$ is the drag coefficient, $S$ is the characteristic square and $m$ is mass.

This method of passive aerodynamic stabilization is described in [1], where some design solutions are describes. Aerodynamic stabilizer is a light inflatable or metal part and can be used for different purposes in the layers of the atmosphere: for aerodynamic stabilization of motion and to obtain the stability of motion of the spacecraft in lower layers of the atmosphere; for preliminary stabilization in the higher layers before the descent; for utilization of space debris by their descent in the highdensity layers of the atmosphere. Aerodynamic stabilizer allows to lower the requirements for characteristics of a spacecraft which might have high mass-inertia and geometrical asymmetry. For the system, the stable motion can be obtained by the proper choice of parameters of the stabilizer.

The conditions for stability of the motion of the system consisting of two solid bodies connected by weightless nonstretchable tether are analyzed in [2], where it is assumed that the system is already deployed.

This research is focused on the deployment process. The mathematical model takes into consideration the extensibility of the tether and allows to evaluate the influence of a method of deployment.

The forms of a spacecraft and a stabilizer are close to spheres, but both of them can have high mass asymmetry. At the initial moment bodies are not separated and move on the circular orbit. The separation takes place with a relatively low relative velocity, and further motion of the bodies depends on the control method which is based on regulation of the release of the tether. The release mechanism works on deceleration only.

\section{Mathematical model of the deployment}

For mathematical modeling the geocentric coordinate system $O x y z$ is used. This coordinate system is connected to the plane of the orbit of the center of mass of the system and is defined at the moment of separation of the stabilizer from the spacecraft. $O x$ axis is directed to the ascending node of the orbit, $O y$ axis is parallel to the vector of velocity of the center of mass at the moment of separation. The spacecraft and the stabilizer are connected by the tether solid bodies (Fig. 1).

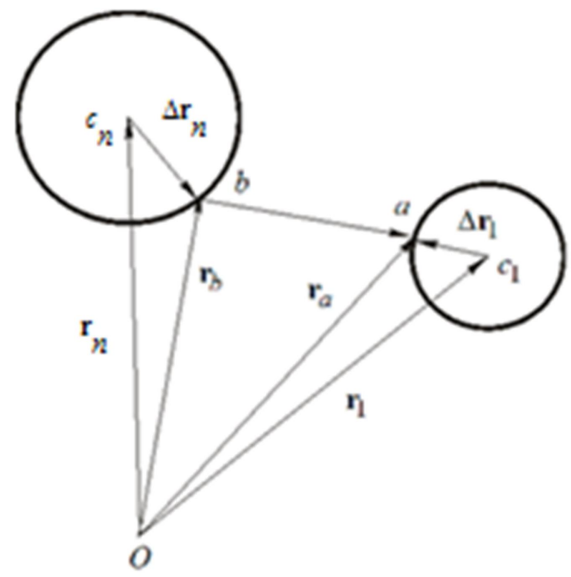

Fig. 1. The scheme of the system. 
The equations of motions of this system are

$$
\begin{aligned}
& m_{i} \frac{d^{2} \mathbf{r}_{i}}{d t^{2}}=\mathbf{G}_{i}+\mathbf{T}_{i}-\mathbf{T}_{i+1}+\mathbf{R}_{i}, i=1,2 \ldots, n \\
& J_{x i} \frac{d \omega_{x i}}{d t}+\omega_{y i} \omega_{z i}\left(J_{z i}-J_{y i}\right)=M_{x i}, J_{y i} \frac{d \omega_{y i}}{d t}+\omega_{x i} \omega_{z i}\left(J_{x i}-J_{z i}\right)=M_{y i}, J_{z i} \frac{d \omega_{z i}}{d t}+\omega_{x i} \omega_{y i}\left(J_{y i}-J_{x i}\right)=M_{z i},
\end{aligned}
$$

where indexes $i=1$ and $i=n$ are for center of masses of a spacecraft and stabilizer respectively, $m_{i}$ are masses and $\mathbf{r}_{i}$ are radius-vectors for bodies and material points on which the tether is divided; $\mathbf{G}_{i}$ and $\mathbf{R}_{i}$ are gravitational and aerodynamic forces, $t$ is time, $\boldsymbol{T}_{i}$ and $\boldsymbol{T}_{i+1} \mathbf{T}_{i+1}$ are acting on adjacent areas of the tether tension forces; $J_{x i}, J_{y i}, J_{z i}$ are moments of inertia of bodies in coordinate systems $c_{i} x_{i} y_{i} z_{i} ; \omega_{x i}, \omega_{y i}, \omega_{z i}$ are projections of angular velocities; $M_{x i}, M_{y i}, M_{z i}$ are projections of acting on each body moments.

During the modeling, gravitational moments are neglected while aerodynamic moments and moments from tension force are taken into consideration. Tension forces are defined by Hooke's law

$$
\mathbf{T}_{i}=T_{i} \frac{\mathbf{r}_{i+1}-\mathbf{r}_{i}}{\left|\mathbf{r}_{i+1}-\mathbf{r}\right|}
$$

where $T_{i}$ is the value of the tension force number $i$. If $\left|\boldsymbol{r}_{i+1}-\boldsymbol{r}\right|$ is less or equal to non-deformed length of the tether on the area number $i$, than the tension force is equal to zero. The spacecraft and the stabilizer are influenced by the tension force from one area of the tether only. For calculation of these forces vectors $\boldsymbol{r}_{a}$ и $\boldsymbol{r}_{b}$ are used. If the tether is not strained, than the free motion of bodies and material point of the tether takes place.

To define gravitational forces, the central gravitational model under Newton's law is used. For the tether, aerodynamic forces are calculated as forces acting on the cylinder [3]. These forces are distributed proportionally between material points of the tether. It is assumed that the motion of the systems takes place in low density gas and the hypothesis of diffuse reflection of gas molecules can be used. [3].

The equations for dynamics of the tether release mechanism are

$$
m_{u} \frac{d^{2} L}{d t^{2}}=T_{1}-F_{u}
$$

where the constant coefficient $m_{u}$ depict the inertia of the tether release mechanism, $F_{u}$ is the control force, $T_{1}$ is the tension force on the first area of the tether, starting from the spacecraft. The tether release mechanism works on deceleration only, $F_{u}>0$, and cannot pull the tether in.

The stabilizer is being separated from the spacecraft with relative velocity $\mathbf{V}_{r}$, and it is necessary to re-calculate velocities basing on the law of impulse saving

$$
\mathbf{V}_{1}^{(a)}=\mathbf{V}_{c}^{(a)}-\frac{m_{2}}{m_{1}+m_{2}} \mathbf{V}_{r}, \quad \mathbf{V}_{2}^{(a)}=\mathbf{V}_{1}^{(a)}+\mathbf{V}_{r},
$$

where $\mathbf{V}_{c}^{(a)}$ is the absolute velocity of the center of mass of the system before the separation, $\mathbf{V}_{1}^{(a)}$ and $\mathbf{V}_{2}^{(a)}$ are absolute velocities after the separation.

\section{The deployment of a tether system and the regulation of the tether release}

During the deployment process dynamic or kinematic control laws can be used. For example, the following dynamic law is used with areas of acceleration and deceleration

$$
F_{p}=\left\{\begin{array}{c}
F_{\text {min }}, t<t_{1} \\
F_{\text {min }}+\left(F_{\text {max }}-F_{\text {min }}\right) \sin ^{2}\left[k_{p}\left(t-t_{1}\right)\right], t_{1} \leq t \leq t_{2}, \\
F_{\text {max }}, t>t_{2}
\end{array}\right.
$$

where $t_{1,2}=t_{p} \pm \pi / 4 k_{p}, t_{p}, k_{p}, F_{\min }, F_{\max }$ are parameters of control law. The switching of control force is made on time bases, here $t_{p}$ - is the time then the force switches. Parameter $k_{p}>0$ defines the smoothness of switching, the lower is it, the smoother is the switching. The parameters of law (4) are defined basing on edge conditions for the end of deployment: $L_{p}\left(t_{k}\right)$, $L_{p}\left(t_{k}\right)=L_{p}\left(t_{k}\right)=0$, where $t_{k}$ is the time of the finishing the deployment.

The dynamic law (4) can be realized using the feedback principle:

$$
F_{u}=F_{p}(t)+p_{L}\left[L-L_{p}(t)\right]+p_{V}\left[L-L_{p}(t)\right],
$$

where $L_{p}(t)$ and $L_{p}(t)$ are program, or nominal, dependencies of length and rate of change of the length of the tether.; $p_{L}, p_{V}$ are feedback coefficients; $L, L$ are perturbed length and rate of change of the length which meet the conditions $(3) ; F_{p}(t)$ is the program decelerating force. 
The principle of regulating on bases of changing of the length and the rate of change of the length of the tether (5) was used in the real orbital tether experiment YES2 [4] and other researches [5,6].

To calculate the control force (5) it is necessary to define the dependencies $F_{p}(t), L_{p}(t), L_{p}(t)$, which can be found numerical by solving the system [5]. According to this, it is required to make prior calculations for these values and to use interpolation during the regulation process. But it is also possible to use more simple principle based on kinematic control law

$$
L_{p}(\tau)=V_{\max } \cos ^{2}(\tau+v)
$$

where $V_{\max }, \omega$ and $v$ are parameters. These parameters are defined from the system of non-linear equations

$$
L_{p}\left(\tau_{k}\right)=0, \quad \frac{d L_{p}}{d \tau}\left(\tau_{k}\right)=0, \quad L_{p}(0)=V_{r}, \int_{0}^{\tau_{k}} L_{p}(\tau) d \tau=L_{k},
$$

where $\tau_{k}=\omega t_{k}$ is dimensionless duration of the deployment.

\section{Numerical results}

The modeling of the deployment of the tether system was made using equations (1-3) and tether release laws (4) and (6). During the release of the tether, the algorithm for inserting the material point was used. This algorithm is described in [5]. On adding new point of the tether, the velocity of a spacecraft and a point of the tether are recalculated basing on the law of conservation of impulse of the system. The relative velocity of a new point is being calculated basing on the relative velocity of the previous point. Relative velocities for the points are defined relatively to the spacecraft. The next results are made for the following initial data: the masses of the spacecraft and the stabilizer are $200 \mathrm{~kg}$ and $20 \mathrm{~kg}$, the final length of the tether is $0.5 \mathrm{~km}$, initial altitude of a circular orbit is $250 \mathrm{~km}$, the linear density of the tether is $0.2 \mathrm{~kg} / \mathrm{km}$, rigidity of the tether is $7000 \mathrm{~N}$, initial relative velocity of separation is $2 \mathrm{~m} / \mathrm{s}$, feedback coefficients $p_{L}=0.2, p_{V}=7.8$. The number of material points for modeling the tether is eight. The task of finding the optimal feedback coefficients was not taken into consideration, and the values of these coefficients were chosen on the assumption of obtaining the stability of regulation processes under the initial perturbations on initial velocity of separation $(25 \%)$ and the direction of separation $( \pm 1 \mathrm{rad})$.

The analysis of numerical results shows that kinematic deployment law (6) has great advantages compared to dynamic law (4). The advantages are based on smoother deceleration of the tether while conditions (7) are used. Usage of dynamic law (4) enforces to solve complex boundary-value problem using numerical calculation for the system of differential equations.

Figure 2 depicts the nominal dependence of rate of change of the tether length $L_{p}(t)$ for this example. Figure 3 shows how the system reacts on the error in velocity of separation equal to $0.5 \mathrm{~m} / \mathrm{s}$. Figure 4 illustrates the dependence of the angle between longitudinal axis of the spacecraft and the direction of the tether $\psi(t)$ from time. This dependence has high importance because it is a condition for exception of sagging and entanglement of the tether.

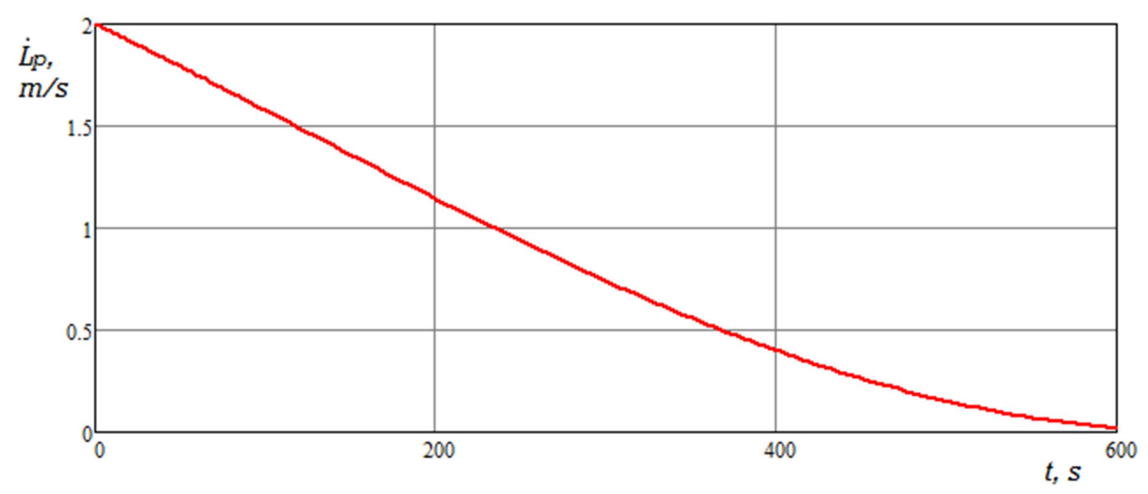

Fig. 2. Nominal dependence of rate of change of the tether length.

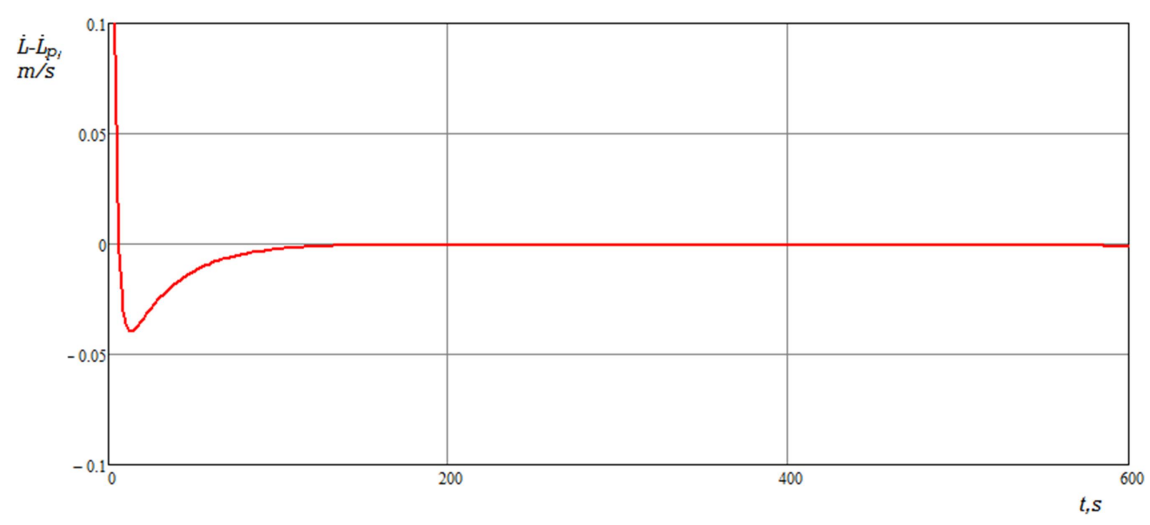

Fig. 3. Reaction on the error in velocity of separation. 


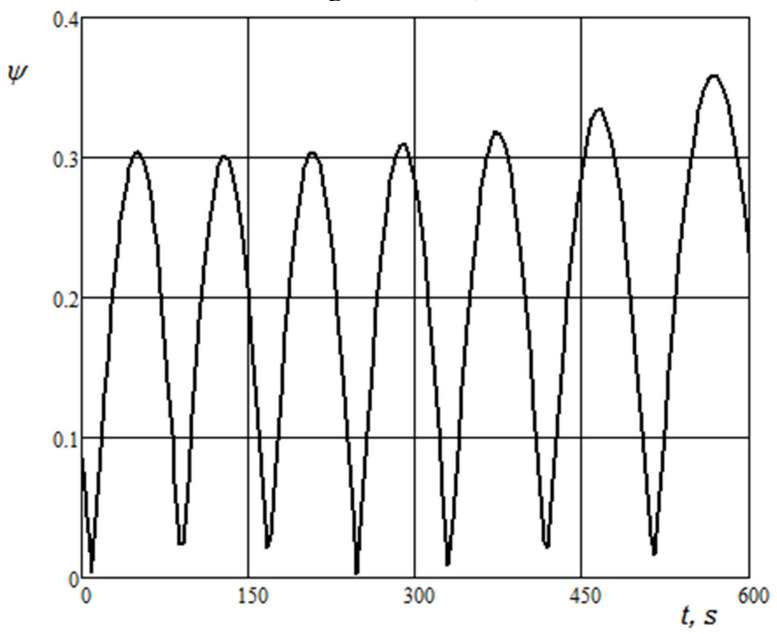

Fig. 4. The dependence of the angle between longitidinal axis of a spaceraft and the tether.

\section{Conclusion}

The analysis of different methods of deployment depicts that dynamics of motion of the system is mainly affected by the moment from tether tension force and almost is not influenced by the static stability of bodies, which is calculated as length between the center of mass and the center of pressure from aerodynamic forces. It is necessary to pay attention to the fact that this research was made for a system with a light and relatively short $0.5 \mathrm{~km}$ tether. The usage of a longer tether leads to increase of an aerodynamic pressure and to the necessity of dividing the tether into larger number of parts during calculations. This means that more computer resources are needed for calculations. Because of this the methods of high performance parallel calculations for analysis of deployment and optimization of parameters of the tether systems are being researched now.

This research is supported by the grant of Russian Foundation for Basic Research (RFBR) 16-41-630637.

\section{References}

[1] Alekseev KB, Bebenin GG. Spacecraft control. Moscow: Mashinostroenie, 1974; 343 p.

[2] Zabolotnov YuM, Elenev DV. Stability of motion of two rigid bodies connected by a cable in the atmosphere. Mechanics of solids 2013; 48(2): 156164. DOI 10.3103/S0025654413020064.

[3] Arzhanikov NS, Sadekova. GS. Aircraft aerodynamics. Moscow: High School, 1983; 360 p.

[4] Kruijff M. Tethers in Space. Netherlands: Delta-Utec Space Research, 2011; 423 p.

[5] Zabolotnov YuM. Control of the deployment of a tethered orbital system with a small load into a vertical position. J. of Applied Mathematics and Mechanics 2015; 79(1): 28-34.

[6] Williams P, Hyslop A, Kruijff M. Deployment control for the YES2 Tether-assisted Re-entry Mission. Advance in the Astronautical Sciences 2006; 123(2): 1101-1120. 